\title{
A Comparison of the Clinical Effectiveness Between Low-Dose Strong Opioids and Non-Steroidal Anti-Inflammatory Drugs in the Treatment of Mild Cancer Pain: A Randomized Trial
}

\author{
Mei-Zuo Liu' \\ Jian $\mathrm{Ma}^{2}$ \\ Jun-Dong $\mathrm{Li}^{1}$ \\ Jing Sun ${ }^{\prime}$ \\ Hua Zhou' \\ Shuo Guan' \\ Ying $\mathrm{Han}^{1}$ \\ Xia Zhang ${ }^{3}$ \\ Ji-Lai Bian' \\ 'Department of Oncology, The Fifth \\ People's Hospital of Dalian, Dalian, \\ I I6000, People's Republic of China; \\ ${ }^{2}$ Intensive Care Unit, The Fifth People's \\ Hospital of Dalian, Dalian, II6000, \\ People's Republic of China; ${ }^{3}$ Medical \\ Examination Center, The Fifth People's \\ Hospital of Dalian, Dalian, II6000, \\ People's Republic of China
}

\begin{abstract}
Objective: The present study aims to explore the effectiveness and safety of low-dose strong opioids compared with non-steroidal anti-inflammatory drugs (NSAIDs) in the treatment of mild cancer pain.

Methods: From September 2016 to September 2018, 66 patients with a malignant tumor and mild cancer pain admitted to the Department of Oncology of Dalian Fifth People's Hospital were divided into the group A (treated with ibuprofen sustained-release tablets for pain relief) and the group B (treated with oxycodone hydrochloride sustained-release tablets for pain relief). After 7 days of treatment, the pain relief (Numeric Rating Scale [NRS]), physical strength, quality of life scores (Zubrod/ECOG/WHO [ZPS]), the Edmonton Symptom Assessment System [ESAS], and the European Organization for Research and Treatment of Cancer Quality of Life Questionnaire-Core15-Palliative [EORTC QLQ-C15PAL] scores), and the occurrence of adverse reactions between the two groups were compared. The occurrence of adverse reactions in the mid-term (after one month and three months of treatment) between the two groups were also compared.
\end{abstract}

Results: Both groups had over $90 \%$ analgesic efficiency, but complete pain relief was more likely to be obtained in the group B $(41.18 \%)$. The total analgesic efficiency in the group B was higher $(100 \%)$ than in the group A $(98.9 \%)$, and the difference was statistically significant $(\mathrm{P}<0.05)$. The differences in the physical strength and quality of life scores in the two groups before and after treatment were statistically significant $(\mathrm{P}<0.05)$. The differences in the ZPS scores between the two groups were statistically significant $(\mathrm{P}<0.05)$. The differences in ESAS and EORTC QLQ-C15-PAL scores between groups were not statistically significant $(\mathrm{P}>0.05)$.

Conclusion: The application of low-dose oxycodone hydrochloride sustained-release tablets as the initial medication for patients with mild cancer pain was safe and effective, and the adverse reactions were easy to manage.

Keywords: oxycodone hydrochloride sustained-release tablet, non-steroidal antiinflammatory drugs, mild cancer pain, clinical effect

\section{Introduction}

At least $1 / 3$ of patients newly diagnosed with a malignant tumor and $70-90 \%$ of patients with advanced tumors have pain. ${ }^{1}$ Pain can directly affect the quality of life in patients with malignant tumors. Patients with cancer pain often have limited activities, sadness, anxiety, and clinical depression, along with suicidal tendencies. 
Their quality of life is significantly lower than in patients with cancer but lacking pain.

In 1982, the World Health Organization (WHO) established a three-step cancer pain treatment program, with the goal of "making cancer patients pain-free by the year 2000". In 2012, the European Society for Medical Oncology (ESMO) recommended low-dose strong opioids or a combination of non-opioid analgesics as an alternative to weak opioids in mild to moderate cancer pain management. ${ }^{2}$ However, in the current clinical practice for cancer pain, most patients with mild pain have difficulty receiving early treatment with strong opioids due to the fact that some clinicians may not thoroughly understand the WHO pain relief principles or adopt the latest treatment options. There is a fear of drug addiction among patients, families, or even doctors due to insufficient knowledge about the drugs, the relatively strict control, and the difficulty in prescribing. This has become the weakest area of palliative care in China. ${ }^{3,4}$

At present, for mild cancer pain, non-steroidal antiinflammatory drugs (NSAIDs) and acetaminophen are often used clinically as first-step drugs. The National Comprehensive Cancer Network (NCCN) guidelines for adult cancer pain ${ }^{5}$ mention caution with the application of NSAIDs, especially in patients on long-term administration, and many oncology patients are at high risk for renal disease, gastrointestinal (GI) tract risk (upper GI surgery, radiation therapy), cardiotoxicity, thrombocytopenia, and bleeding disorders. The FDA warns that the long-term application of NSAIDs increases the risk of heart disease and stroke. It is important to note in clinical practice that the potential adverse reactions of chemotherapy (especially the anti-angiogenic inhibitors), such as hematologic (thrombocytopenia or coagulopathy), renal, hepatic, and cardiovascular system toxicity, can increase with the concomitant application of NSAIDs.

Attention should be paid to those with a high risk for the use of NSAIDs. These included those with an increased risk of renal toxicity: patients aged $>60$ years, with fluid imbalance, multiple myeloma, diabetes mellitus, interstitial nephritis, renal papillary necrosis, and patients with concomitant use of other nephrotoxic agents (including cyclosporine and cisplatin) and chemotherapeutic agents that are metabolized by the kidneys. Those with an increased risk of GI tract toxicity: patients aged $>60$ years, with peptic ulcer or history of alcohol abuse (3 or more alcoholic beverages per day), patients with vital organ dysfunction (including hepatic failure), patients on long-term high-dose NSAIDs, on a combination of steroids, or daily cardioprotective doses of aspirin. Those with a high risk of cardiotoxicity: patients with a history of cardiovascular disease or increased risk for cardiovascular disease or complications. For many patients, opioids are a safe and effective alternative analgesic to NSAIDs.

Oxycodone hydrochloride sustained-release tablets are pure agonists of opioid receptors, with an affinity for both the $\mu$ and $\kappa$ receptors, no ceiling effect, and no upper limit for dose in the application. The adverse reactions are mainly dizziness and drowsiness associated with its role in the central nervous system, and constipation, nausea, vomiting, and urinary difficulties associated with increased smooth muscle tone of the GI tract. Rare adverse reactions to opioids are respiratory depression; these occur primarily with acute drug poisoning, which is rare in the use of common oral sustained-release formulations in clinical practice. In the drugs' actual clinical application, we find that the abovementioned adverse reactions are time-limited and can be self-resolved, except constipation, which requires long-term attention. In terms of cardiovascular adverse reactions, therapeutic opioids only mildly reduce myocardial oxygen consumption and left ventricular end-diastolic pressure. ${ }^{6}$ In addition, the morphine analogs can mimic the protective effect of ischemic preadaptation against the myocardial ischemic injury, reducing the area of infarction and decreasing myocardial cell death. The kidney mainly metabolizes opioids in the form of morphine-6-glucuronide, and slow excretion may occur in older patients or those with reduced renal function, ${ }^{7,8}$ which requires appropriate attention in clinical application. Opioids are not hepatotoxic, and are safe in clinical practice.

In summary, the NCCN guidelines suggest that NSAIDs should be used appropriately in patients with mild cancer pain and advocate the early application of opioids.

Based on the relatively good control of moderate to severe cancer pain in China, 66 patients with mild cancer pain admitted to our hospital were selected. The present study aims to provide the clinical basis for the early application of strong opioids and achieve early analgesia for patients with cancer pain.

\section{Materials and Methods Participants}

From September 2016 to September 2018, the clinical data of patients who were diagnosed with malignant tumors and 
mild cancer pain in the Department of Oncology at Dalian Fifth People's Hospital were collected by our research team. The inclusion criteria were: (1) patients with tumors confirmed by clinical and/or pathological diagnosis; (2) patients aged $\geq 18$ years; (3) patients with tumor-related somatic or visceral pain; (4) patients with a score on the Numeric Rating Scale (NRS) for pain <4 points; (5) patients who were opioid-naive (it is considered by the Food and Drug Administration [FDA] that patients with opioid tolerance are those who receive at least $60 \mathrm{mg}$ of morphine daily, at least $30 \mathrm{mg}$ of oxycodone daily, or other equivalent opioids for one week or longer); (6) patients who could take oral medication and could be excluded from the effect of antineoplastic therapy (eg, radiotherapy, chemotherapy, targeted therapy) on analgesia; (7) patients with expected survival time $>3$ months. The exclusion criteria were: (1) patients who were unable to take oral medication; (2) patients who were treated with strong opioids before the present study; (3) patients with neuropathic pain; (4) patients who were unable to perform and cooperate with the follow-up visits; (5) patients with contraindications to opioids, including hypoxic respiratory depression, head injury, paralytic intestinal obstruction, acute abdomen, delayed gastric emptying, chronic obstructive respiratory disease, pulmonary heart disease, acute or severe bronchial asthma, hypercapnia, allergy to oxycodone, moderate to severe liver dysfunction, severe renal dysfunction (creatinine clearance $<10 \mathrm{~mL} / \mathrm{min}$ ), chronic constipation, taking monoamine oxidase inhibitors, and discontinuing monoamine oxidase inhibitors for less than 2 weeks; (6) patients who were unsuitable for the present study, according to the judgment of the investigators, for any reasons other than the inclusion and exclusion criteria, including allergy to ibuprofen, aspirin or other non-steroidal anti-inflammatory drugs, a history of peptic ulcer, gastrointestinal bleeding or perforation, taking non-steroidal antiinflammatory drugs before this study, and acute pulmonary embolism. A total of 66 validated cases were included in the analysis.

This single-center, randomized, single-blinded of subjects clinical study was conducted with approval from the Ethics Committee of The Fifth People's Hospital of Dalian. This study was conducted in accordance with the declaration of Helsinki. Written informed consent was obtained from all participants.

\section{Medication Regimen}

In the group $\mathrm{A}$, the first-step drug ibuprofen sustainedrelease tablets (300mg orally, twice daily) were given to treat pain. In the group B, low-dose strong opioid analgesic treatment with oxycodone hydrochloride sustainedrelease tablets was given for pain (10 mg orally every twelve hours as the starting dose, and the dose was adjusted according to the pain status of the patient), 7 days of continuous dosing.

The condition of pain control was observed at 4, 12, 24, 48, and 72 hours after administration, and pain control, life improvement, and occurrence of adverse reactions were followed up for seven days and occurrence of adverse reactions in each group in the mid-term (one month and three months).

\section{Evaluation Methods}

Before starting the study, each patient and their family members were educated about pain medication and the rating scales by an experienced pain clinician. These included (1) pain assessment: NRS was adopted for pain assessment at the beginning of and during the treatment. Comprehensive assessment of pain: Patients were assessed for pain on admission using the Brief Pain Assessment Scale and were dynamically assessed daily after pain management. (2) Functional strength score: functional strength assessment using the Zubrod/ECOG (Eastern Cooperative Oncology Group)/WHO (ZPS) 5-point scale was conducted before treatment, when the patient reached a stable state of pain relief, and weekly thereafter. (3) Quality of life assessment: The European Organization for Research and Treatment of Cancer Quality of Life Questionnaire-Core15-Palliative (EORTC QLQ-C15PAL) scale and The Edmonton Symptom Assessment System (ESAS) were used to assess the change in the quality of life in patients with cancer pain before and after treatment.

\section{Observation and Recording Indicators}

(1) The same pain clinician collected the general characteristics of all patients: age, gender, type of cancer, cause of pain, pain location and duration, the score data of NRS, ZPS, ESAS, and EORTC QLQ-C15-PAL before and during treatment were recorded. (2) The same pain clinician calculated the degree of pain relief after 7 days of treatment. Pain control was defined as: no relief $=$ no reduction in pain intensity (NRS scores) compared with the baseline; partial relief $=$ reduce $30 \%$, but not up to $50 \%$ in pain intensity (NRS scores) compared with the baseline; significant relief $=$ reduce $50 \%$, but not up to $100 \%$ in pain intensity (NRS scores) compared with the baseline; complete relief $=100 \%$ 
reduction in pain intensity (NRS scores) compared with the baseline. Total analgesic efficacy $=$ (number of cases with partial pain relief + significant pain relief + complete pain relief)/total number of cases $\times 100 \%$. (3) Observation of the adverse reactions of the drug: the adverse reactions during the application of analgesic drugs were observed and recorded by our research team and attending doctors of patients, and the degree of adverse reactions were recorded, according to the adverse drug reaction classification criteria in the Common Terminology Criteria for Adverse Events (CTCAE) version 4.0.

\section{Sample Size Calculation}

The sample size was calculated based on the effective rate of analgesia. It is estimated that the effective rate of analgesia in group A (treated with ibuprofen sustainedrelease tablets) is about $50 \%$. The effective rate of analgesia in group B (treated with oxycodone hydrochloride sustained-release tablets) was $100 \%$. Set $\alpha=0.05$ (bilateral), and power $=0.90$. The PASS 11 software was used to calculate the sample size, which was 12 cases in each group. Assuming that the loss to follow-up rate of the study is $20 \%$, the sample size needed for each group is 15. Finally, 34 patients were included in the treatment group of oxycodone hydrochloride sustained-release tablets, and 32 patients were included in the treatment group of ibuprofen sustained-release tablets.

\section{Statistical Methods}

The SPSS 23.0 software was adopted for statistical analysis. Measurement data were tested by the $t$-test and expressed as $\bar{x} \pm s$. The $\mathrm{X}^{2}$ test was used for countable data. $\mathrm{P}<0.05$ was considered statistically significant.

\section{Results}

\section{General Characteristics}

Sixty-seven cases were included in the present study and the patients were divided into 34 cases in group B and 33 cases in group A by random number table method. One patient in group A was not continued into the group due to acute pulmonary embolism. Finally, 66 cases were included in this study, 34 cases in group B and 32 cases in group A (Figure 1). There were 34 males and 32 females, six in stage III and 60 in stage IV cancer. There were no statistically significant differences between the two groups in terms of age, gender, tumor stage, and nature of pain $(\mathrm{P}>0.05)$ (Table 1).

\section{Pain Relief, Physical Strength and Quality of Life Scores Between the Two Groups Primary Endpoint: Comparison of the Pain Relief Between the Two Groups}

Patients in group B (treated with oxycodone hydrochloride sustained-release tablets) had no outbreak pain and the dose of the drug was $10 \mathrm{mg}$ orally every 12 hours. The analgesic efficacy in both groups was over $90 \%$, but the group B patients were more likely to obtain complete pain relief (41.18\%) and achieve a completely pain-free life. When comparing the analgesic effects between the two groups, the total analgesic efficacy in the group B was higher than in the group $\mathrm{A}$, and the difference was statistically significant $(\mathrm{P}<0.05)$ (Table 2$)$.

\section{Secondary endpoint: Comparison of the physical strength and quality of life scores between the two groups.}

The differences in the ZPS scores before and after treatment in the group $\mathrm{A}$ and $\mathrm{B}$ were statistically significant $(\mathrm{P}<0.05)$. Before treatment, there was no statistically significant difference in the ZPS score between the two groups $(\mathrm{P}>0.05)$. After treatment, the difference in the ZPS score was statistically significant between the two groups; the improvement in the group B was superior to that in the group A $(\mathrm{P}<0.05)$. In terms of quality of life, the differences in the ESAS and EORTC QLQ-C15-PAL scores before and after treatment were statistically significant in both groups $(\mathrm{P}<0.05)$, but the differences between groups were not statistically significant $(\mathrm{P}>0.05)$ (Tables 3-table 5).

\section{Comparison of the Adverse Reactions}

The adverse reactions in both groups were focused on nausea, constipation, dizziness, drowsiness, stomach ache, and elevated transaminases; one patient in the group B had difficulty with urination. The above adverse reactions were degrees I-II according to the CTCAE v4.0 and could be relieved by symptomatic treatment, and no serious adverse reactions of degree III or above occurred. The incidence of dizziness, nausea, and constipation was higher in the group B. Constipation could be controlled by laxative drugs such as lactulose and soft jaggery capsules (A laxative drugs that is a stool softener), while dizziness and nausea resolved themselves after three to five days of tolerance. The incidence of stomach ache and transaminase elevation was higher in the group A. There was no statistically significant difference in the incidence of nausea, vomiting, dizziness, drowsiness, dysuria, constipation, stomach pain, and 


\section{CONSORT}

TRANSPARENT REPORTING of TRIALS

\section{CONSORT 2010 Flow Diagram}

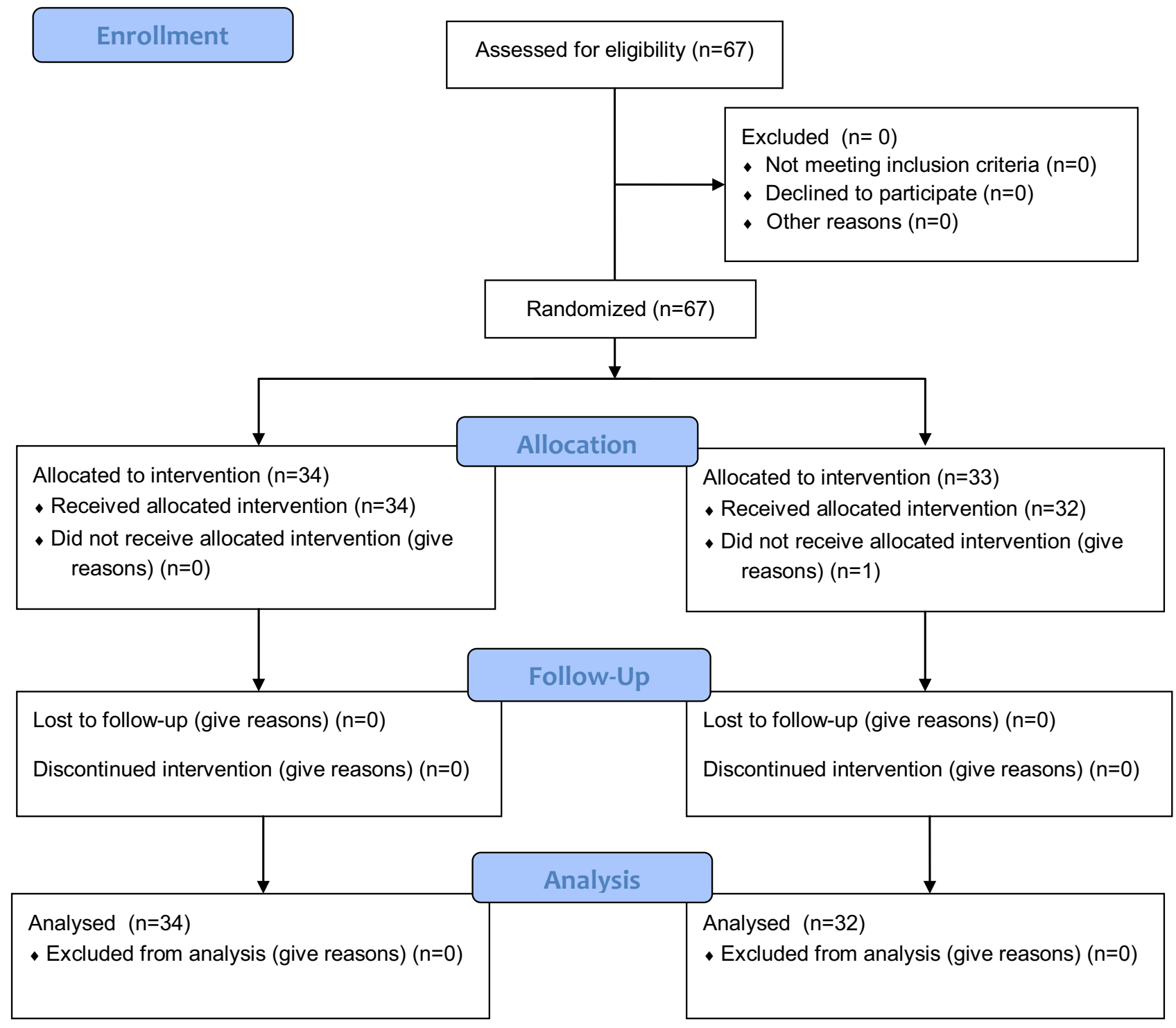

Figure I Study flow diagram and follow-up.

transaminase elevation in the group B compared with the group A ( $>$ > 0.05) (Table 6). In this trial, ibuprofen sustained-release tablets were taken orally for 7 consecutive days. After the mid-term observation of adverse reactions (after one month and three months of treatment), no adverse reactions, such as gastrointestinal bleeding, kidney injury or myocardial injury, were observed. Therefore, they were not included in data analysis. 
Table I Comparison of General Clinical Data Between Two Groups of Patients

\begin{tabular}{|c|c|c|c|c|}
\hline Items & Group A (Ibuprofen) & Group B (Oxycodon) & t / X2 Value & $P$ value \\
\hline $\mathrm{n}$ & 32 & 34 & & \\
\hline Age & $62.88 \pm 2.27$ & $63.94 \pm 2.25$ & 0.333 & 0.74 \\
\hline \multicolumn{5}{|l|}{ Gender } \\
\hline Male & 17 & 17 & 0.064 & 0.8 \\
\hline Female & 15 & 17 & & \\
\hline \multicolumn{5}{|l|}{ Staging } \\
\hline III & 3 & 3 & 0.006 & 0.938 \\
\hline IV & 29 & 31 & & \\
\hline \multicolumn{5}{|l|}{ Types of Cancer } \\
\hline Lung Cancer & 21 & 19 & 1.118 & 0.777 \\
\hline Breast Cancer & I & 2 & & \\
\hline Digestive tract tumors & 6 & 9 & & \\
\hline Others & 4 & 4 & & \\
\hline \multicolumn{5}{|l|}{ Causes of pain } \\
\hline Disease Related & 28 & 31 & 0.235 & 0.705 \\
\hline Treatment Related & 4 & 3 & & \\
\hline \multicolumn{5}{|l|}{ Pain Location } \\
\hline Chest & II & II & 6.557 & 0.239 \\
\hline Abdomen & 6 & 10 & & \\
\hline Back & 5 & 5 & & \\
\hline Limbs & 7 & 5 & & \\
\hline Bone & 3 & 3 & & \\
\hline \multicolumn{5}{|l|}{ Nature of pain } \\
\hline Dull pain & 17 & 18 & 1.37 & 0.788 \\
\hline Soreness and pain & 9 & 10 & & \\
\hline Compressive-like pain & 2 & 4 & & \\
\hline Others & 4 & 2 & & \\
\hline Duration of pain & $26.44 \pm 33.625$ & $26.03 \pm 32.793$ & 0.013 & 0.908 \\
\hline
\end{tabular}

Table 2 Comparison of Analgesic Effects Between the Two Groups

\begin{tabular}{|l|c|c|c|c|c|}
\hline Groups & Complete Relief & Significant Relief & Partial Relief & No Relief & Total Analgesic Efficiency \\
\hline Group A (Ibuprofen) & 2 & 11 & 18 & 1 & $98.9 \%$ \\
Group B (Oxycodon) & 14 & 13 & 7 & 0 & $100 \%$ \\
X2 & & & & 15.254 \\
P value & & & & 0.001 \\
\hline
\end{tabular}

Table 3 Comparison of ZPS Scores Before and After Treatment Between the Two Groups

\begin{tabular}{|l|c|c|c|c|}
\hline ZPS & Before Treatment & After Treatment & t Value & P value \\
\hline Group A (Ibuprofen) & $1.56 \pm 0.669$ & $1.00 \pm 0.568$ & 3.626 & $0.00 \mathrm{I}$ \\
Group B (Oxycodon) & $1.50 \pm 0.663$ & $0.65 \pm 0.597$ & 5.575 & 0.000 \\
t value & -0.381 & -2.457 & & \\
P value & 0.704 & 0.017 & & \\
\hline
\end{tabular}


Table 4 Comparison of Edmonton Symptom Assessment Scale Before and After Treatment Between the Two Groups

\begin{tabular}{|l|c|c|c|c|}
\hline ESAS & Before Treatment & After Treatment & t Value & P value \\
\hline Group A (lbuprofen) & $40.34 \pm 6.025$ & $20.19 \pm 4.299$ & 33.449 & 0.000 \\
Group B (Oxycodon) & $40.00 \pm 7.224$ & $18.82 \pm 5.589$ & 35.163 & 0.000 \\
t value & -0.209 & -1.106 & & \\
P value & 0.835 & 0.273 & & \\
\hline
\end{tabular}

Table 5 Comparison of EORTC QLQ-CI5-PAL Quality of Life Scores Before and After Treatment Between the Two Groups

\begin{tabular}{|l|c|c|c|c|}
\hline EORTC QLQ-CI5-PAL & Before Treatment & After Treatment & t Value & P value \\
\hline Group A (Ibuprofen) & $33.06 \pm 3.95 I$ & $21.09 \pm 2.374$ & 20.536 & 0.000 \\
Group B (Oxycodon) & $32.44 \pm 4.73$ & $20.00 \pm 2.686$ & 20.325 & 0.000 \\
t value & -0.577 & -1.749 & & \\
P value & 0.566 & 0.085 & & \\
\hline
\end{tabular}

Table 6 Comparison of the Incidence of Adverse Reactions Between the Two Groups

\begin{tabular}{|l|c|c|c|c|}
\hline Items & Group A (Ibuprofen) & Group B (Oxycodon) & X2 Value & P value \\
\hline Nausea & $0(0)$ & $3(8.8)$ & 2.958 & 0.239 \\
Dizziness & $4(I 2.5)$ & $10(29.4)$ & 2.821 & 0.134 \\
Drowsiness & $0(0)$ & $3(8.8)$ & 2.958 & 0.239 \\
Difficulty in urination & $0(0)$ & $I(2.9)$ & 0.956 & 1.00 \\
Constipation & $3(9.4)$ & $9(26.5)$ & 3.239 & 0.11 \\
Stomach ache & $10(31.3)$ & $5(14.7)$ & 2.569 & 0.145 \\
Elevated transaminases & $7(21.9)$ & $2(5.9)$ & 3.58 & 0.079 \\
\hline
\end{tabular}

\section{Discussion}

Pain is one of the most common clinical symptoms in patients with cancer, seriously threatening physical and mental health, quality of life. WHO prepared and published the "Cancer Pain Relief" guidelines in 1986 and put forward the three-step pain treatment principles: stepwise administration, oral administration, timely administration, individualized administration, and attention to specific details. The stepwise administration refers to the administration of NSAIDs \pm adjuvants for mild cancer pain; weak opioids \pm non-opioid analgesics or adjuvants for moderate cancer pain; and strong opioids \pm non-opioid analgesics or adjuvants for severe cancer pain. ${ }^{9}$ Since 1990, China has been promoting the WHO three-step pain relief principle for the clinical treatment of cancer pain nationwide. After 30 years of clinical practice in China, pain control in patients with cancer pain has significantly improved. ${ }^{10}$ Since 2017, China has put forward a new concept for cancer pain treatment, from effective pain control to advocating early pain control. A study in patients with moderate to severe cancer pain using oxycodone hydrochloride sustained-release tablets as a background found that the 12-hour rapid adjustment of the background dose could achieve a rapid pain improvement. ${ }^{11}$

However, patients with mild cancer pain with NRS $<4$ points are a special group whose pain symptoms are less severe than those with moderate to severe cancer pain, and their willingness for treatment is not strong. The neglected early pain interventions may cause resistance to subsequent antitumor therapy in these patients, and the symptoms of pain affect the quality of life and cause unnecessary suffering. Therefore, early intervention for pain management and good long-term control is an urgent need for clinical treatment. Currently, NSAIDs are often used for pain management in patients with mild pain. However, if cancer pain is not effectively relieved, it is difficult to get complete pain relief, so most patients still have to face long-term pain relief treatment.

In a randomized trial, 100 patients with mild to moderate cancer pain were divided into a conventional WHO threestep analgesic treatment group and an immediate strong opioid treatment group. The treatment satisfaction was 
compared between the two groups. Patients treated early with strong opioids had better pain control, a 2.61 point reduction in the average visual analogue score (VAS), and a significant improvement in quality of life compared with patients treated on the traditional WHO three-step treatment. Patients treated with strong opioids had higher treatment satisfaction, satisfactory overall improvement in pain intensity, and fewer changes in treatment. Early intervention for cancer pain significantly improves quality of life, prolongs survival, increases treatment satisfaction, and lowers health care costs. Similar results have also been obtained from several other trials. $^{12-16}$

The results of the present study showed that for patients with mild cancer pain, both low-dose oxycodone hydrochloride sustained-release tablets and first-step drugs could improve pain, but low-dose oxycodone hydrochloride extended-release tablets were significantly more effective than first-step drugs $(41.18 \%$ vs $0.06 \%)$ and a completely pain-free life, and improving the quality of life compliance with future antitumor therapy. In terms of adverse reactions, nausea, constipation, dizziness, and drowsiness in both groups were relieved with symptomatic treatment. However, the incidence of dizziness and constipation was higher in the oxycodone hydrochloride sustained-release tablet group, while the incidence of stomach ache and elevated transaminases were higher in the firststep drug treatment group.

In conclusion, in selecting drugs for clinical application, the location of the primary tumor, the estimated duration of pain control, the level of the GI mucosal barrier, and the status of hepatic and renal function should be taken into consideration. The application of low-dose oxycodone hydrochloride sustained-release tablets as the initial medication for patients with mild cancer pain might be safe and effective, and the adverse effects might be easy to grasp and handle, so it is worthy of further clinical application to improve quality of life and treatment tolerance. The present study was a small sample case-control study. A large sample randomized controlled study is needed to obtain more reliable evidence-based medical evidence to guide clinical application.

\section{Funding}

Dalian Medical Science Research Program Project Funding (1811061).

\section{Disclosure}

The authors report no conflicts of interest in this work.

\section{References}

1. Kapil R, Nolting A, Roy P, Fiske W, Benedek I, Abramowitz W. Pharmacokinetic properties of combination oxycodone plus racemic ibuprofen: two randomized, open-label, crossover studies in healthy adult volunteers. Clin Ther. 2004;26(12):2015-2025. doi:10.1016/j. clinthera.2004.12.013

2. Ripamonti CI, Santini D, Maranzano E, Berti M, Roila F; ESMO Guidelines Working Group. Management of cancer pain: ESMO Clinical Practice Guidelines. Ann Oncol. 2012;23 Suppl 7:vii13954. doi:10.1093/annonc/mds233

3. Kaasa S. Is cancer pain control improved by a simple who pain analgesic ladder approach combined with tumor-directed treatment? J Clin Oncol. 2016;34(5):399-400. doi:10.1200/JCO.2015.64.7537

4. Cleary J, Silbermann M, Scholten W, Radbruch L, Torode J, Cherny NI Formulary availability and regulatory barriers to accessibility of opioids for cancer pain in the Middle East: a report from the Global Opioid Policy Initiative (GOPI). Ann Oncol. 2013;24 Suppl 11:xi51-9.

5. Swarm RA, Paice JA, Anghelescu DL, et al. Adult Cancer Pain, Version 3.2019, NCCN Clinical Practice Guidelines in Oncology. J Natl Compr Canc Netw. 2019;17(8):977-1007. doi:10.6004/ jnccn.2019.0038

6. National Health and Family Planning Commission Rational Drug Use Expert Committee, China Pharmacists Association. Guide to the Rational Use of Drugs for Coronary Heart Disease (2nd edition). Chine J Front Med. 2018;10(6):1-130.

7. Hamamoto-Hardman BD, Steffey EP, Weiner D, McKemie DS, Kass P, Knych HK. Pharmacokinetics and selected pharmacodynamics of morphine and its active metabolites in horses after intravenous administration of four doses. $J$ Vet Pharmacol Ther. 2019;42 (4):401-410. doi:10.1111/jvp.12759

8. King S, Forbes K, Hanks GW, Ferro CJ, Chambers EJ. A systematic review of the use of opioid medication for those with moderate to severe cancer pain and renal impairment: a European Palliative Care Research Collaborative opioid guidelines project. Palliat Med. 2011;25(5):525-552. doi:10.1177/0269216311406313

9. WHO. Cancer Pain Relief. Geneva, Switzerland: WHO; 1986.

10. Li TD. The past, present and future of cancer pain control at home and abroad. Qi Lu Zhong Liu Za Zhi. 1997;3:4-6.

11. Guo J, Lv J, Liu B, Xing LG, Xiao J, Wang ZH. Effect of hydrochloride oxycodone controlled-release tablets with dose titration for moderate or severe cancer pain. Chine J Cancer Control. 2017;24 (18):1319-1322.

12. Marinangeli F, Ciccozzi A, Leonardis M, et al. Use of strong opioids in advanced cancer pain: a randomized trial. J Pain Symptom Manage. 2004;27(5):409-416. doi:10.1016/j.jpainsymman.2003.10.006

13. Temel JS, Greer JA, Muzikansky A, et al. Early palliative care for patients with metastatic non-small-cell lung cancer. $N$ Engl J Med. 2010;363(8):733-742. doi:10.1056/NEJMoa1000678

14. Baker H. Low-dose morphine for moderate cancer pain. Lancet Oncol. 2016;17(2):e47. doi:10.1016/S1470-2045(15)00608-7

15. Bandieri E, Romero M, Ripamonti CI, et al. Early Strong Opioid Treatment Study (ESOT) Investigators. Randomized Trial of Low-Dose Morphine Versus Weak Opioids in Moderate Cancer Pain. $J$ Clin Oncol. 2016;34(5):436-442. doi:10.1200/ JCO.2015.61.0733

16. Uekuzu Y, Higashiguchi T, Futamura A, et al. Study on Administration of Opioid Antagonists in Terminal Cancer Patients: 7 Patients Receiving Opioid Antagonists Following Opioids among 2443 Terminal Cancer Patients Receiving Opioids. Biol Pharm Bull. 2017;40(3):278-283. doi:10.1248/bpb.b16-00715 


\section{Publish your work in this journal}

The Journal of Pain Research is an international, peer reviewed, open access, online journal that welcomes laboratory and clinical findings in the fields of pain research and the prevention and management of pain. Original research, reviews, symposium reports, hypothesis formation and commentaries are all considered for publication. The manuscript management system is completely online and includes a very quick and fair peer-review system, which is all easy to use. Visit http:// www.dovepress.com/testimonials.php to read real quotes from published authors.

Submit your manuscript here: https://www.dovepress.com/journal-of-pain-research-journal 